\title{
Melanoma Brain Metastases: Local Therapies, Targeted Therapies, Immune Checkpoint Inhibitors and Their Combinations-Chances and Challenges
}

\author{
Marvin Kuske ${ }^{1,2,3} \cdot$ Ricarda Rauschenberg $^{1,2,3} \cdot$ Marlene Garzarolli $^{1,2,3}$. \\ Michelle Meredyth-Stewart ${ }^{4}$ - Stefan Beissert ${ }^{1,2,3}$ - Esther G. C. Troost $^{3,5,6,7,8}$. \\ Oliva Isabella Claudia Glitza ${ }^{9}$. Friedegund Meier $^{1,2,3}{ }_{D}$
}

Published online: 7 February 2018

(C) The Author(s) 2018. This article is an open access publication

\begin{abstract}
Recent phase II trials have shown that BRAF/ MEK inhibitors and immune checkpoint inhibitors are active in patients with melanoma brain metastases (MBM), reporting intracranial disease control rates of $50-75 \%$. Furthermore, retrospective analyses suggest that combining stereotactic radiosurgery with immune checkpoint inhibitors or BRAF/MEK inhibitors prolongs overall survival. These data stress the need for inter- and multidisciplinary cooperation that takes into account the individual prognostic factors in order to establish the best treatment for
\end{abstract}

Friedegund Meier

Friedegund.Meier@uniklinikum-dresden.de

1 Department of Dermatology, Medical Faculty of Technische Universität Dresden, University Hospital Carl Gustav Carus, University of Dresden, Fetscherstr. 74, 01307 Dresden, Germany

2 Skin Cancer Center at the University Cancer Centre Dresden, Dresden, Germany

3 National Center for Tumor Diseases (NCT), Partner Site Dresden, Dresden, Germany

4 Department of Internal Medicine, Medical Faculty of Technische Universität Dresden, University Hospital Carl Gustav Carus, Dresden, Germany

5 Department of Radiation Oncology, Medical Faculty Carl Gustav Carus, University Hospital, Technische Universität Dresden, Dresden, Germany

6 OncoRay-National Center for Radiation Research in Oncology, Dresden, Germany

7 Dresden and German Cancer Research Center (DKFZ), German Cancer Consortium (DKTK), Heidelberg, Germany

8 Institute of Radiooncology, Helmholtz-Zentrum DresdenRossendorf, Dresden, Germany

9 Department of Melanoma Medical Oncology, The University of Texas MD Anderson Cancer Center, Houston, TX, USA each patient. Although the management of MBM has dramatically improved, a substantial number of patients still progress and die from brain metastases. Therefore, there is an urgent need for prospective studies in patients with MBM that focus on treatment combinations and sequences, new treatment strategies, and biomarkers of treatment response. Moreover, further research is needed to decipher brain-specific mechanisms of therapy resistance.

\section{Key Points}

Recent studies report encouraging results for BRAF/ MEK inhibitors and anti-PD-1/anti-CTLA-4 antibodies in the treatment of patients with melanoma brain metastases (MBM). However, a substantial number of patients still progress and die from brain metastases.

Retrospective studies suggest an overall survival benefit with acceptable toxicity for stereotactic ablative radiotherapy combined with BRAF/MEK inhibitors or immune checkpoint inhibitors.

Treatment decisions for patients with MBM should be made by an interdisciplinary tumor board in order to establish the best possible treatment for the individual patient.

For patients with MBM, further research on brainspecific mechanisms of therapy resistance and prospective clinical studies are essential. 


\section{Introduction}

Melanomas are the third most common source of cerebral metastases, preceded only by non-small-cell lung cancer (NSCLC) and breast carcinomas [1]. The propensity of malignant melanomas to metastasize into the central nervous system (CNS) becomes clear considering that the incidence of malignant melanoma is far lower than that of NSCLC or breast cancer. Therefore, melanoma has the highest tendency to metastasize to the brain. The risk of brain metastases in metastatic melanoma increases with disease duration. Melanoma brain metastases (MBM) have been identified in up to $75 \%$ of metastasized melanoma patients at autopsy [2].

Prognostic factors are important not only for the choice of treatment, but also for assessing and comparing research results. Retrospective studies have demonstrated that the survival rates of patients with MBM are correlated to the number of cerebral metastases, the presence of neurological symptoms, the serum level of LDH (lactate dehydrogenase), the patient's age, the simultaneous presence of extracerebral metastases, the BRAF status (with positive BRAF mutation status being associated with a good prognosis) and the patient's physical condition [3-6].

The spectrum of available treatments for metastatic melanoma has increased substantially over the last 6 years due to the approval of effective immunotherapies and targeted treatments. However, until recently, patients with brain metastases have been excluded from most clinical studies, and prognoses remained poor, with survival typically measured in a few months if untreated [7, 8]. Luckily, however, this has changed. The phase II studies conducted to date have demonstrated activity of new systemic therapies in MBM and have therefore improved the prognosis for patients with MBM. However, most patients with MBM still progress and die, which stresses the urgency of further studies and research to improve the outcome of these patients.

The aim of this review is to provide an update on the treatment options for $\mathrm{MBM}$, including the most recent research results.

\section{Local Treatments}

To date, local treatment modalities such as neurosurgical resection, stereotactic radiosurgery (SRS), stereotactic ablative radiotherapy (SABR), or whole brain radiotherapy (WBRT) have been the mainstays of treatment of brain metastases.

\subsection{Neurosurgical Resection}

Neurosurgical resection is indicated for patients with either large lesions that cannot be safely irradiated with SRS/ SABR or for symptomatic lesions, particularly when fast improvement of neurological symptoms can be achieved by removal. Another indication for surgery is tissue retrieval for histological analysis in patients without history of primary tumors. However, the metastases have to be accessible and the patient's general condition adequate to undergo surgery.

The largest retrospective study conducted on the efficiency of local treatment of patients with MBM to date evaluated the data of 686 patients [7]. The median overall survival (mOS) for patients having undergone neurosurgical resection (8.7 months) or neurosurgical resection with adjuvant radiotherapy (8.9 months) was significantly longer than that of patients having received radiotherapy alone (3.4 months) or best supportive care (2.1 months) $(p<0.001)$. Notably, most patients received WBRT, with a minority of $7.9 \%$ of the patients having been treated with SABR, occasionally combined with WBRT.

\subsection{Stereotactic Ablative Radiotherapy and Radiosurgery}

In SABR, an intense dose of high-energy photons is applied to a circumscribed lesion, while sparing the healthy surrounding tissue. A special form of SABR is SRS, in which the total dose of irradiation is delivered in one single fraction. In general, SRS/SABR is carried out in patients with three to four metastases measuring $<3$ to $4 \mathrm{~cm}$ in $1-6$ fractions [9, 10]. In a prospective study on 1194 patients, Yamamoto et al. [11] treated up to 10 brain metastases (total cumulative volume $\leq 15 \mathrm{~mL}$ ) of different histologies using SRS. The SRS-related toxicity in the patient cohort with five to ten brain metastases was similar to that of the cohort with only two to four brain metastases. Interestingly, patients with one brain metastasis had a statistically significant better overall survival ( $p=0.0004)$, whereas median survival of the cohort with two to four metastases was non-statistically different from that of the cohort with five to ten metastases $(p=0.78)$. It seems that the total volume of cerebral metastases influences outcome rather than the number of lesions [12].

The reported 12-month local control rates after SRS for MBM range from 52 to $75 \%$ [13, 14]. Large and hemorrhagic metastases are adversely affecting local control [13]. The mOS of patients with MBM following SRS has been reported to be 5-11 months [9, 13, 14]. Markers for better prognosis were found to be good performance status, a single brain metastasis, and lack of extracerebral visceral metastases $[15,16]$. 
The incidence of side effects following SRS/SABR is $2-14 \%$, and includes headache, nausea, vomiting, and seizures resulting from edema and hemorrhage [17-19]. One major complication, which in general may occur several months (usually $8-11$ months, in rare case$\mathrm{s}<3$ months) or years after irradiation and is associated with nonspecific symptoms, is radionecrosis [20]. Pathologically, radionecrosis is a coagulation necrosis resulting from chronic inflammation, with dysfunction of the endothelial cells, enhanced capillary permeability, and finally secondary vascular occlusion of small vessels [20].

Treatment options include high doses of corticosteroids, the anti-VEGF antibody bevacizumab, and surgical resection [21, 22].

\subsection{Whole Brain Radiotherapy (WBRT)}

To date, WBRT has been employed in patients with multiple (symptomatic) brain metastases, including those arising from metastasized melanoma. With total doses of 20-30 Gy administered over the course of 1-2 weeks, the mOS is approximately 3 months [7, 23].

The decision for or against WBRT should in particular take into account its acute, but also its late side effects; for example, fatigue, alopecia, xerostomia, and neurocognitive deficits, which negatively impact on quality of life [24]. Poor neurocognitive function before the delivery of WBRT may predict clinical deterioration [25]. The advantage of WBRT over best supportive care alone has not yet been investigated in randomized controlled studies. The authors of the Cochrane Collaboration Review [26] concluded that in patients with brain metastases, advanced (systemically uncontrolled) disease, and poor general condition, palliative treatment without WBRT may be appropriate in some cases.

However, in patients with multiple brain metastases or leptomeningeal metastases, for whom SRS or neurosurgical resection is not indicated, WBRT combined with new systemic therapies may play an increasingly important role. Teng et al. [27] found an increase in the permeability of the blood-tumor barrier in brain metastases that had previously shown low permeability in 30 patients (53\% melanoma patients) 2-4 weeks after WBRT. However, prospective clinical trials are needed to confirm that these findings ultimately will translate into clinical efficacy.

Furthermore, hippocampus-sparing WBRT may decrease deterioration in neurocognitive performance. One study in 113 patients showed a significantly smaller decline in cognitive performance 4 months after treatment with hippocampus-sparing WBRT than in a historical comparison group having received non-hippocampus-sparing WBRT [28]. Several prospective studies including extensive neurocognitive function tests are currently accruing patients.

\subsection{Adjuvant Radiotherapy Following Surgery}

Following surgical resection of MBM, adjuvant radiotherapy (WBRT or SRS/SABR) to the resection cavity can be considered to lower the risk of local recurrence. In a prospective phase III clinical trial with 128 patients with brain metastases (21\% melanoma patients), SRS to the surgical cavity significantly lowered local recurrence compared with that noted for observation alone in patients with one to three completely resected brain metastases [29]. A meta-analysis with eight retrospective cohort studies including a total of 646 patients with brain metastases (melanoma patients being a minority) compared the efficacy of post-operative SRS/SABR (234 patients) to post-operative WBRT (408 patients). The number of local recurrences was similar irrespective of the radiotherapy technique. When compared with SRS/SABR, however, WBRT seemed to offer better protection from leptomeningeal metastases with improved overall survival, albeit not reaching statistical significance [30].

One multicenter retrospective study demonstrated that in patients with MBM who were treated with surgery or SABR ( $n=200$ patients), the addition of WBRT prolonged overall survival (9 months [95\% CI 8-14] vs 7 months [95\% CI 6-9]; $p=0.0458$ ) [4]. However, other studies failed to show longer survival with adjuvant radiotherapy (WBRT or SABR) following neurosurgical resection [31].

In a randomized study of 95 patients with brain metastases (two with MBM), post-operative WBRT after resection of a single metastasis $(n=49)$ resulted in less frequent recurrence of the tumor anywhere in the brain (18 vs $70 \% ; p<0.001)$ than did no further treatment for the brain metastases $(n=46)$. Although there was no statistically significant difference between the two groups regarding overall survival, patients who received WBRT were less likely to die from neurological causes than were patients without WBRT [32].

\subsection{Adjuvant WBRT Following Stereotactic Radiosurgery (SRS)}

Similar to adjuvant WBRT following brain metastases resection, adjuvant WBRT following SRS appears to increase local control of treated lesions, decrease new sites of brain metastases, and decrease the need for salvage SRS or WBRT [18, 31, 33]. However, adjuvant WBRT following SRS has not been shown to improve overall survival [18, 31, 33]. 
A phase III trial investigated the role of WBRT after SRS or neurosurgery for one to three brain metastases. The 359 patients with brain metastases (18 melanoma patients) who had undergone surgery $(n=160)$ or SRS $(n=199)$ were randomized to no further intervention or WBRT (30 Gy in 10 fractions) [31]. The addition of WBRT to the post-surgical management reduced the 2-year relapse rate both at the initially treated sites and at other sites in the brain. Although no improvement in overall survival was found, this phase III trial demonstrated that adjuvant WBRT lowered the number of neurologic deaths. This stresses the importance of appropriate patient selection. A subgroup of patients with multiple MBM and high risk to die from neurological causes might benefit from adjuvant WBRT. This subgroup has to be characterized more deeply.

\subsection{Conclusions on Local Therapy}

In summary, patient selection plays a key role in successful local therapy. The best candidates for neurosurgical resection of brain metastases are patients with a very limited number of superficial metastases in non-eloquent areas of the brain and adequate condition to undergo surgery. This is especially the case when fast symptom relief is needed, and patients do not have a history of primary tumors. Patients suitable for SRS may have up to ten metastases with a total cumulative volume $\leq 15 \mathrm{~mL}$. The role of (adjuvant) WBRT after surgery or SRS has been questioned. It seems to be a treatment option when patients have a high risk to die from neurological causes; however, the potentially detrimental effects on neurocognitive function and quality of life should be considered.

\section{Systemic Therapies}

\subsection{Cytotoxic Chemotherapy}

Prior to the marketing approval of ipilimumab and vemurafenib, the alkylating agent dacarbazine (DTIC) was considered to be the standard treatment for patients with non-resectable melanoma metastases. Alternative therapies were temozolomide, a derivative of DTIC that permeates the blood-brain barrier, and fotemustine, a nitrosourea alkylating agent, neither of which had a significant effect on overall survival [9].

\subsection{Targeted Therapy}

The serine-threonine kinase BRAF and the G-protein NRAS are integral components of the MAP kinase signal transduction pathway. Approximately $40 \%$ of cutaneous melanomas exhibit activating BRAF mutations and 15-25\% exhibit activating NRAS (neuroblastoma rat sarcoma virus) mutations [34]. Combination therapies consisting of a BRAF inhibitor (dabrafenib or vemurafenib) and an MEK inhibitor (trametinib or cobimetinib) have been approved for systemic treatment of BRAF-V600 mutated melanoma since 2015. The COMBI-D and coBRIM phase III studies have shown that combination therapy with BRAF and MEK inhibitors were superior to single agent BRAF inhibitor, leading to a significant improvement in response (with response rates of 69 vs 53 and 69.6 vs $50 \%$, respectively), median progression-free survival (mPFS) (11 vs 8.8 and 12.3 vs 7.2 months, respectively) and mOS (25.1 vs 18.7 and 22.3 vs 17.4 months, respectively) [35, 36] [37]. Patients with active brain metastases were excluded from these studies.

Table 1 details the two prospective phase II studies investigating BRAF inhibitor monotherapy in patients with MBM, the BREAK-MB study [38] and the study by McArthur et al. [39]. In the BREAK-MB study [38], 172 patients with asymptomatic brain metastases and BRAF V600E or BRAF V600 K mutation were treated with dabrafenib. For patients with BRAF V600E-mutant melanoma and untreated brain metastases, the intracranial response rate $(\mathrm{ICR}=\mathrm{PR}$ [partial response] $+\mathrm{CR}$ [complete response]) was 39\%, and the mOS was 7.6 months. For patients with BRAF V600E-mutant melanoma and prior local brain therapy, the ICR was $31 \%$, and the mOS was 7.2 months. In the phase II study by McArthur et al. [39], 146 patients with BRAF V600-mutant melanoma and brain metastases were treated with vemurafenib. For the entire cohort, the ICR was $18 \%$, and the mOS was 8.9-9.6 months. In this study, the patients exhibited several unfavorable prognostic factors, such as symptomatic MBM, four or more brain metastases in 16\%, an Eastern Cooperative Oncology Group (ECOG) of 0 in only 43\%, and elevated LDH levels in $55 \%$ of patients.

In the COMBI-MB phase II study, patients with BRAFV600-mutant metastatic melanoma and MBM were treated with the BRAF inhibitor dabrafenib in combination with the MEK inhibitor trametinib in the approved dose. A maximum of two previous systemic therapies was allowed, but no previous treatment with a BRAF or MEK inhibitor [40]. Patients were assigned into four cohorts. Cohort A included patients with asymptomatic MBM without previous local treatment, cohort B consisted of patients with asymptomatic MBM after prior local therapy, cohort C comprised patients with BRAFV600D/K/R mutations, and cohort D included patients with symptomatic MBM. Comparing the results of the different cohorts, one has to keep in mind that the percentage of enhanced LDH and the cohort size were different between the cohorts (see also Table 1). The ICR for patients with asymptomatic MBM 


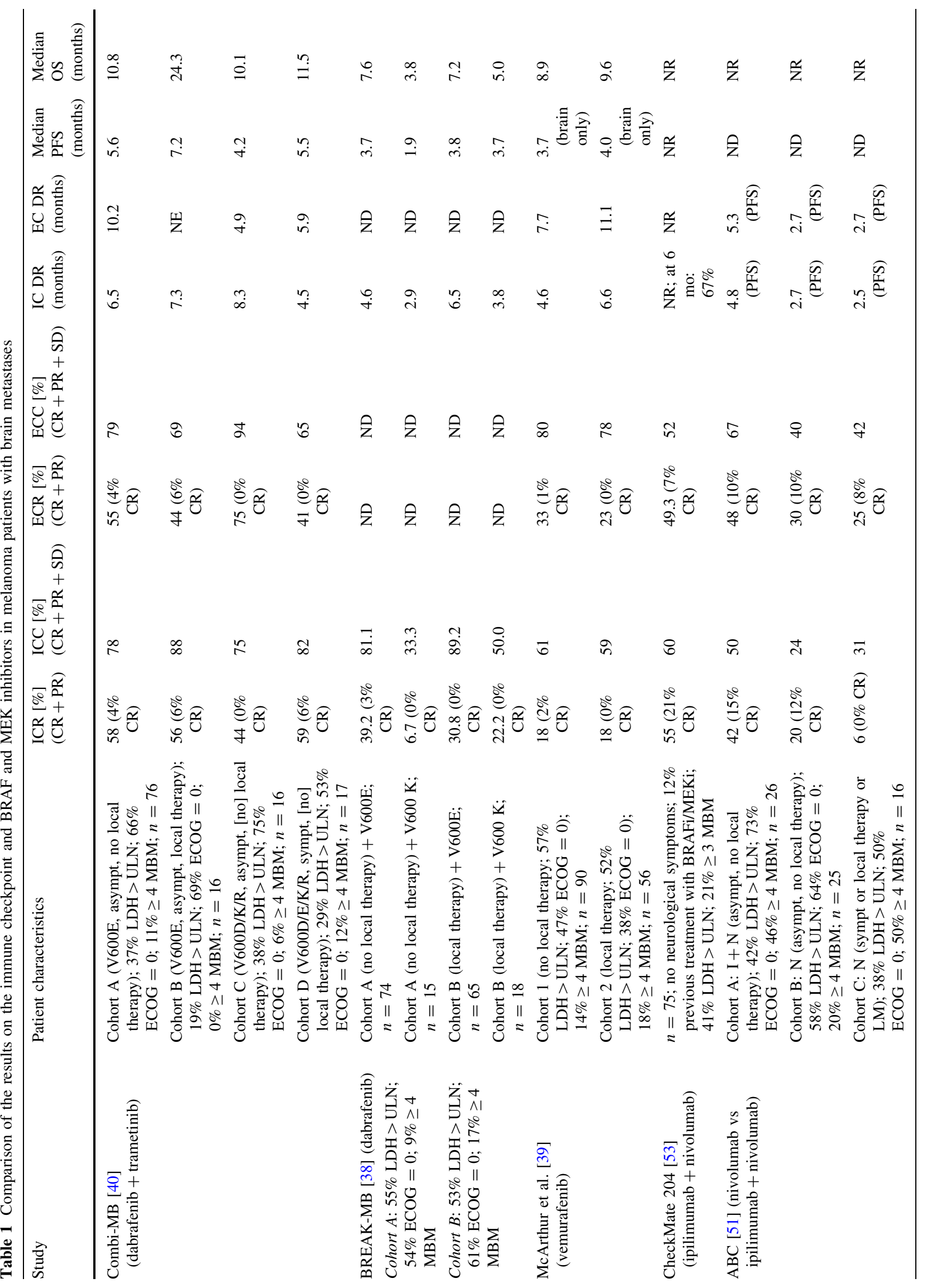




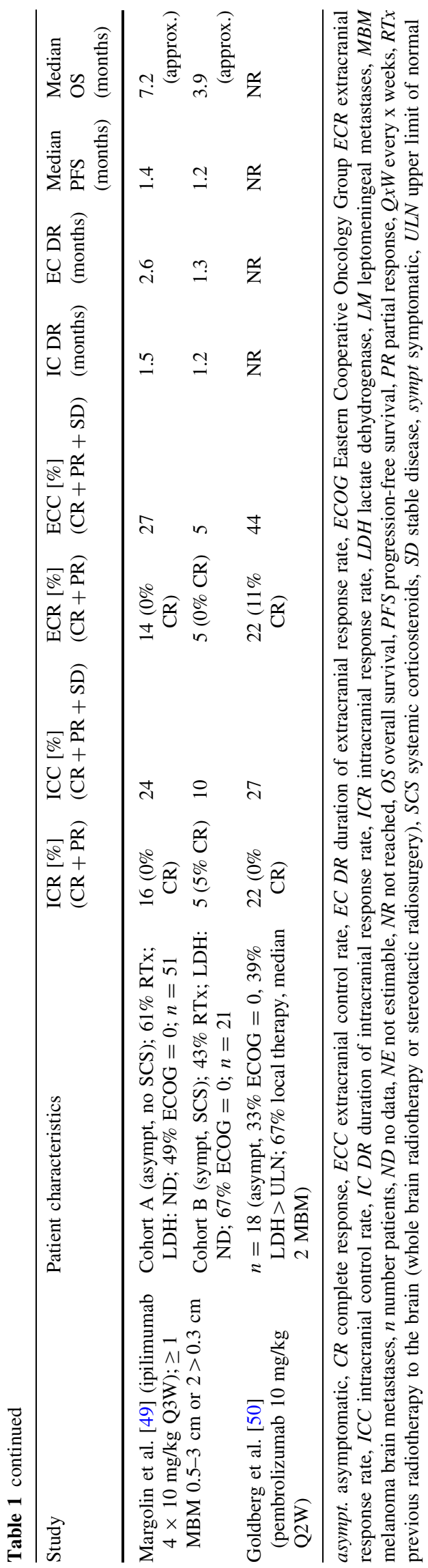

without prior local treatment (cohort A) was 58\%. Interestingly, the ICR for patients with symptomatic MBM in cohort $\mathrm{D}$ was $59 \%$. The median duration of intracranial response for patients with asymptomatic MBM not having received prior local treatment was 6.5 months. The mPFS and mOS for patients with asymptomatic MBM not having received prior local treatment were 5.6 and 10.8 months, respectively. Moreover, cohort $\mathrm{B}$, which included asymptomatic patients following prior local treatment, showed a distinctly better result than the other groups, with an mPFS of 7.2 months and an mOS of 24.3 months (see also Table 1). These data suggest that local therapy such as radiotherapy before systemic treatment may prolong survival. However, the patient number in this cohort was small $(n=16)$ and most patients ( $81 \%)$ had normal LDH levels. In this study, no unexpected toxicities were observed. A total of $8 \%$ of patients had to terminate the treatment due to adverse events [40].

In the COMBI-MB study, the ICR was comparable to the extracranial response rate. However, the median duration of extracranial response was longer than the median duration of intracranial response (10.2 vs 6.5 months). Interestingly, intracranial progression only was observed in $47 \%$ of patients, whereas extracranial progression only occurred in $9 \%$ of patients. A lack of concordance between intra- and extra-cranial disease control has also been shown in other studies. In a retrospective analysis of 86 patients treated with vemurafenib, $20 \%$ had developed brain metastases on average 5.3 months after vemurafenib initiation. The extracranial disease was well controlled in 59\% of patients during intracranial progression. Death was considered as directly related to intracranial progression in $71 \%$ of patients [41]. These data suggest that there may be brain-specific mechanisms of BRAF/MEK inhibitor resistance, in addition to the primary and secondary resistance mechanisms active in both intra- and extracranial disease processes (for a summary, see [42]).

\subsection{Immunotherapy}

The immune system is capable of combating tumor cells. Due to changes in the immunogenicity of tumors, the antitumor response of the immune system is a dynamic process of immunosurveillance and tumor progression, which can result in the emergence of immune-resistant tumor cell variants [43]. Cancer immunotherapy attempts to stimulate the immune system to destroy immune-resistant tumors.

Hitherto, high-dose interleukin-2 (HD IL-2) was the only approved immunotherapy to modify the natural history of patients with stage IV melanoma and resulted in long-term survival in a small fraction of patients. Reported response $(\mathrm{CR}+\mathrm{PR})$ and $\mathrm{CR}$ rates were 16-18 and 7-8\%, respectively. Furthermore, HD IL-2 appears to be active in 
brain metastases [44]. However, the severe toxicity of HD IL-2 (e.g., capillary leak syndrome and systemic inflammatory response syndrome) limited the application to carefully selected patients treated at centers with experience in managing the side effects [44, 45].

Optimal activation of antigen-specific lymphocytes requires specific antigen recognition by lymphocytes, as well as additional costimulatory signals. Immune checkpoints are costimulatory or coinhibitory receptors of T-cell activation [46]. The development of blocking antibodies against the coinhibitory immune checkpoints CTLA-4 (cytotoxic T-lymphocyte-associated antigen 4; e.g., ipilimumab) or PD-1 (programmed death 1; e.g., nivolumab, pembrolizumab) resulted in remarkable outcomes in patients with unresectable melanoma, with response rates of $58 \%$ for ipilimumab in combination with nivolumab and $44 \%$ for nivolumab alone and overall survival rates at 3 years of $58 \%$ for ipilimumab plus nivolumab and $52 \%$ for nivolumab alone [47, 48]. However, patients with active MBM were excluded from these studies.

Phase II studies demonstrated the efficacy of immune checkpoint inhibitors in patients with MBM. The initial studies with single-agent ipilimumab [49] (see Table 1) and single-agent pembrolizumab [50] were followed by studies combining ipilimumab with nivolumab, based on the results of clinical trials in melanoma patients with systemic disease [47].

In a phase II study, the efficacy of pembrolizumab was tested at a dosage of $10 \mathrm{mg} / \mathrm{kg}$ every 2 weeks in melanoma patients $(n=18)$ with at least one new or progressive asymptomatic brain metastasis measuring between 5 and $20 \mathrm{~mm}$ and an ECOG performance status $\leq 1$. Additional brain metastases that were $>20 \mathrm{~mm}$ or whose location gave cause for concern were treated by means of local therapy (SRS, WBRT, or neurosurgical resection) before inclusion in the study. This was the case for 20 of the total number of 36 patients. The patients had an average of two brain metastases and an ECOG performance status of 0 in $33 \%$ of cases. The ICR was $22 \%$. Patients who responded to treatment showed an ongoing response at the time of the report (ranging from 4 to 10 months). Known grade 3-4 adverse events (Common Terminology Criteria for Adverse Events [CTCAE]) occurred in 14\% of patients and grade 2 headaches in $19 \%$ of patients [50].

In a phase II study, patients with MBM not previously treated with an immune checkpoint inhibitor (prior treatment with a BRAF/MEK inhibitor was permitted) were assigned to three cohorts. In cohort $\mathrm{A}$, patients with asymptomatic MBM and no prior local therapy were treated with nivolumab plus ipilimumab (nivolumab $1 \mathrm{mg}$ / $\mathrm{kg}+$ ipilimumab $3 \mathrm{mg} / \mathrm{kg}$, every 3 weeks [Q3W] $\times 4$, then nivolumab $3 \mathrm{mg} / \mathrm{kg}$ Q2W). In cohort B, patients with asymptomatic MBM and no prior local therapy were treated with nivolumab alone (nivolumab $3 \mathrm{mg} / \mathrm{kg}$ Q2W), and in cohort $\mathrm{C}$ patients with prior treatment and patients with symptomatic MBM or leptomeningeal metastases received nivolumab alone (nivolumab $3 \mathrm{mg} / \mathrm{kg}$ Q2W). In this study, $46 \%$ of patients had more than four MBM, and $42 \%$ of patients had elevated serum levels of LDH. The ICR for cohort A was $42 \%$, for cohort B $20 \%$, and for cohort $\mathrm{C} 6 \%$. The extracranial response rate (ECR) for cohort A was $48 \%$ and thus comparable with the ICR of $42 \%$. For cohort A, the intracranial progression-free survival was 4.8 months, and the extracranial progression-free survival was 5.3 months. The combination therapy with ipilimumab plus nivolumab had a toxicity profile similar to previous reports [47], with $46 \%$ of patients experiencing grade $3 / 4$ adverse events and $27 \%$ of patients discontinuing treatment [51].

A remarkable aspect of this study is that in BRAF/MEK inhibitor pretreated patients, the response rate dropped to $16 \%$.

Interestingly, a recent comparative, transcriptomicmethylomic analysis of patient-matched melanoma metastases biopsied before therapy and during disease progression showed that resistance to targeted therapies is associated with the acquisition of highly recurrent nongenomic alterations as well as changes in the immune landscape of the tumor that may result in cross-resistance to salvage anti-PD-1/PD-L1 immunotherapy [52]. Targeted therapy sequenced or combined with immune checkpoint inhibitors is currently under investigation in several prospective studies.

In a further phase II study [53], the approved dose of nivolumab combined with ipilimumab was investigated in melanoma patients with at least one asymptomatic MBM. Prior treatment with BRAF/MEK inhibitors was permitted. Exclusion criteria included neurological symptoms, steroids for more than 10 days, WBRT, prior treatment with immune checkpoint inhibitors, and leptomeningeal metastases. Seventy-nine percent of patients had one or two MBM. After a median follow-up of 9.2 months, the ICR was $55 \%$, and the extracranial response rate was $49 \%$. Interestingly, $21 \%$ of patients experienced complete remission of MBM. The intracranial progression-free survival rate after 6 months was $67 \%$. The combination therapy with ipilimumab plus nivolumab had a toxicity profile similar to previous reports. However, headaches were reported in $25 \%$ of patients ( $4 \%$ grade $3-4$ ).

\subsection{Symptom Management}

For patients with symptomatic MBM, local treatment options such as neurosurgery should be checked to achieve quick symptom relief [54]. 
Systemic corticosteroids are recommended to provide temporary relief of CNS symptoms related to increased intracranial pressure secondary to brain metastases. Dexamethasone (4-16 mg/day or more) is the corticosteroid of choice, mainly because of its limited mineralocorticoid activity. For patients who are no longer at risk of neurologic compromise, attempts should be made to provide the minimum effective dose of steroids to prevent acute and long-term steroid sequelae. However, tapering should occur slowly over several weeks to avoid rebound symptoms [55].

Control of epileptic seizures by anticonvulsants such as levetiracetam is another important treatment goal for patients with symptomatic MBM [54]. However, there is no evidence to support the prophylactic use of antiepileptic drugs that may cause side effects and drug interactions [56].

\section{Combinations of Systemic and Local Treatments}

Radiotherapy induces immunogenic cell death leading to inflammasome formation and attraction of immune cells [57]. The killed tumor cell becomes a source of new tumor antigens (neoantigens) which have been masked. This 'insitu vaccination' can strengthen a systemic antitumor immune response and may lead to regression of metastases outside the irradiated volume [58]. This phenomenon is called the abscopal effect [59]. Although the abscopal effect appears to be rare, it may be enhanced by combining immunotherapy with radiotherapy, making a rare event clinically relevant $[60,61]$. There is pre-clinical and clinical data that support this hypothesis [62-64]. A few impressive abscopal responses have been reported after radiation of metastases in patients who were unresponsive or had ceased to respond to immunotherapy [65-67].

Combining radiotherapy and BRAF/MEK inhibition can also be expected to have a synergistic effect because BRAF inhibition induces G1 cell cycle arrest. The concomitant decrease of cells in the $\mathrm{S}$ phase is associated with decreased resistance to radiotherapy [68]. In addition, Hecht et al. [69] demonstrated that lymphocytes of patients undergoing vemurafenib therapy were more sensitive to radiation than those of patients not receiving vemurafenib. The radiosensitizing effect of vemurafenib was attributed to an impairment of DNA repair mechanisms.

In a retrospective analysis of 108 patients with MBM having undergone SRS, $73.1 \%$ of patients were given systemic therapy (anti-CTLA4, anti-PD-1, BRAF inhibitor without or with MEK inhibitor) within 6 weeks of SRS. The median brain metastasis control was 7.5 months in patients receiving SRS and ipilimumab, 12.7 months in patients receiving SRS and anti-PD-1 therapy, and
12.7 months in patients receiving SRS and BRAF inhibitors without or with MEK inhibitors. The mOS was 7.5 months in patients treated with SRS and ipilimumab, 20.4 months in patients treated with SRS and anti-PD-1 therapy, and 17.8 months in patients treated with SRS and BRAF inhibitors without or with MEK inhibitors. mOS was 10.8 months for patients who received SRS only; SRS with additional immunotherapy or $\mathrm{BRAF} \pm \mathrm{MEK}$ inhibitor therapy was associated with a longer survival than SRS alone (hazard ratio [HR] 0.51; 95\% CI 0.25-1.05; and HR 0.30 ; 95\% CI 0.14-0.64) [70].

Additional retrospective studies also demonstrate a better outcome for patients with MBM when SRS and immune or targeted therapy are combined [71].

In a prospective phase I study, 16 patients with MBM underwent WBRT ( $\operatorname{arm~A} ; n=5$ ) or SRS ( $\operatorname{arm~B} ; n=11$ ) followed by ipilimumab $3 \mathrm{mg} / \mathrm{kg}(n=7)$ or $10 \mathrm{mg} / \mathrm{kg}$ $(n=9)$ every 3 weeks for four total doses, starting on day 3 of WBRT or 2 days after SRS. Eleven grade 3 toxicities were reported, with gastrointestinal toxicities the most common (31\%). No patient developed grade 4 or 5 toxicity. The mOS was 8 months in arm A and not reached in arm B [72].

In a retrospective analysis, 26 patients with MBM were treated with SRS within 6 months of receiving nivolumab. Seventy-three percent of patients had more than one MBM and $88 \%$ of patients had a Karnofsky performance status of $\leq 90 \%$. The mOS was 11.8 months [73].

In another retrospective study, six patients were treated with SRS within 3 months of dabrafenib and trametinib administration. Three patients $(50 \%)$ had three or more MBM and all patients had a Karnofsky performance status of $\geq 90 \%$. The mOS was 20.0 months. There was no evidence of increased or unexpected toxicity with the two modalities combined [74].

In a further retrospective study, the clinical outcomes of more than 300 patients with MBM were analyzed. Thirtyfive patients had a known BRAF V600E mutation status of their tumors. A total of 191 patients underwent SRS, 22 patients were treated with BRAF inhibitors and 32 patients were treated with PD- $1 \pm$ CTLA- 4 inhibitors. There was a significant reduction in the 12-month cumulative incidence of distant failure of MBM among patients who received BRAF inhibitor treatment after upfront SRS (68 vs 95\%, $p=0.03$ ). On multivariate analyses, BRAF inhibition within 30 days of SRS was protective against local failure (HR $0.08 ; 95 \%$ CI $0.01-0.55 ; p=0.001$ ). There was no increased risk of radionecrosis seen when SRS and BRAF inhibition was combined [75].

The occurrence of cerebral radiation necrosis following SRS in combination with immunotherapy or targeted therapy has been reported [70, 76]. Considering the high incidence of radiation necrosis following SRS alone (up to 
$34 \%$ after 24 months [20]), it is currently not clear whether the incidence of radiation necrosis is increased by immune or targeted therapy. The majority of retrospective analyses revealed that combining SRS with $\mathrm{BRAF} \pm$ MEK inhibitors or immune checkpoint inhibitors does not increase radiotoxicity. Moreover, this combination strategy appears to improve brain metastasis control and to prolong survival [77-82]. However, concurrent radiation treatment and targeted therapy can cause significant skin toxicity. The new consensus guidelines from the ECOG recommend holding BRAF inhibitors $\geq 1$ day before and after SRS and $\geq 3$ days before and after fractionated radiation therapy [83].

The sequencing of and time interval between radiotherapy and systemic therapy may be a de-cisive factor for therapeutic efficacy. In a mouse model, radiotherapy 24 hours after systemic therapy induced up-regulation of PDL1 on tumor cells and PD-1 on T cells. This effect was sustained for at least 7 days. Radiotherapy and anti-PD-1 antibodies administered in short succession may thus enhance the therapeutic effect of radiotherapy [84].

Qian et al. [85] analyzed the data of 75 patients with MBM who had received SRS and anti-CTLA4 $(n=54$; $72 \%)$ or anti-PD1 $(n=21 ; 28 \%)$. SRS within 4 weeks of the first or last dose of immunotherapy significantly improved the response rates of MBM after 1.5, 3, and 6 months. A trend towards longer mOS was found for patients treated with immunotherapy within 4 weeks of SRS $(p=0.0691 ; 19.1$ months [range 2.7-64.2] vs 9.0 months [range 2.1-61.8 months]).

Furthermore, preclinical studies suggest that fractionation of radiotherapy and dose per fraction may play an important role in eliciting an abscopal effect. Dewan et al. [64] demonstrated that fractionated but not single-dose radiotherapy in combination with anti-CTLA-4 antibody induces an abscopal effect, suggesting that pulsatile antigen exposure may be important to mediate this effect. Highdose fractionated radiation therapy is considered to trigger pro-inflammatory immune responses, whereas low-dose fractionated radiation therapy may kill infiltrating CD8+ T cells over time (summarized in $[57,86]$ ). However, further clarification is needed to define the most appropriate fractionation to be used in combination with immunotherapy.

\section{Leptomeningeal Metastases}

Melanoma patients with leptomeningeal metastases (LM) face the worst prognosis, with an mOS of 8-10 weeks, despite systemic treatment (fotemustine and temozolomide) and/or intrathecal chemotherapy (methotrexate, liposomal cytarabine, thiotepa) and/or radiotherapy [5, 87-89].
Melanoma is among the solid tumors with the highest rates of LM, with a reported incidence of 5-25\% [90]. The diagnosis of LM can be challenging. Clinical symptoms can be subtle, and include headache, cognitive changes, disturbances of consciousness, stiff neck, hemiparesis, paresis of the facial nerve, and ataxia. Work-up includes T1-weighted contrast enhancement in computed magnetic resonance images. However, the sensitivity depends on the technique of examination and the experience of the radiologist, ranging from 36.4 to $81.8 \%$ [91]. Examination of the cerebrospinal fluid (CSF) with identification of tumor cells has a high specificity ( $>95 \%)$. However, the first lumbar puncture has low sensitivity $(<50 \%)$. Repeated lumbar puncture increases the sensitivity up to $80 \%$ [87, 90, 92, 93].

Case reports suggest that BRAF/MEK inhibitors and immune checkpoint inhibitors can be effective in patients with LM. Some case reports even report long-term survival [94-97]. In a retrospective analysis of 39 melanoma patients with LM, the mOS of patients having received therapy (BRAF/MEK inhibitors, ipilimumab, radiation) was significantly longer than the mOS of untreated patients (16.9 vs 2.9 weeks, $p<0.001$ ) [98]. In a retrospective case series [99], melanoma patients with LM treated with antiPD-1 antibodies and/or BRAF inhibitors with or without radiotherapy had an mOS of 5.2 months.

Papadopoulos et al. treated 46 melanoma patients with LM with intrathecal interleukin-2 (IL-2). In this study, treatment response was defined as normalization of CSF cytology for at least 4 weeks. The responders (34\%) had an mOS of 11.5 months compared with the non-responders with an mOS of 3.5 months. Adverse events included fever, chills, changes in mental status and increased intracranial pressure. However, no treatment-related deaths were reported [100]. Further case reports confirm the feasibility, potential benefit, and manageable toxicity of intrathecal IL-2 therapy [101]. Furthermore, successful intrathecal administration of tumor infiltrating lymphocytes has been reported [102] (ClinicalTrials.gov; NCT00338377).

In view of the rising incidence of LM, prospective studies in patients with LM are urgently needed. Currently, a phase II trial of pembrolizumab (NCT03091478) and a phase II trial of ipilimumab and nivolumab (NCT02939300) in melanoma patients with LM are open to recruitment.

\section{Algorithm for the Management of Melanoma Brain Metastases}

A pragmatic algorithm for the management of melanoma brain metastases is proposed in Fig. 1. 


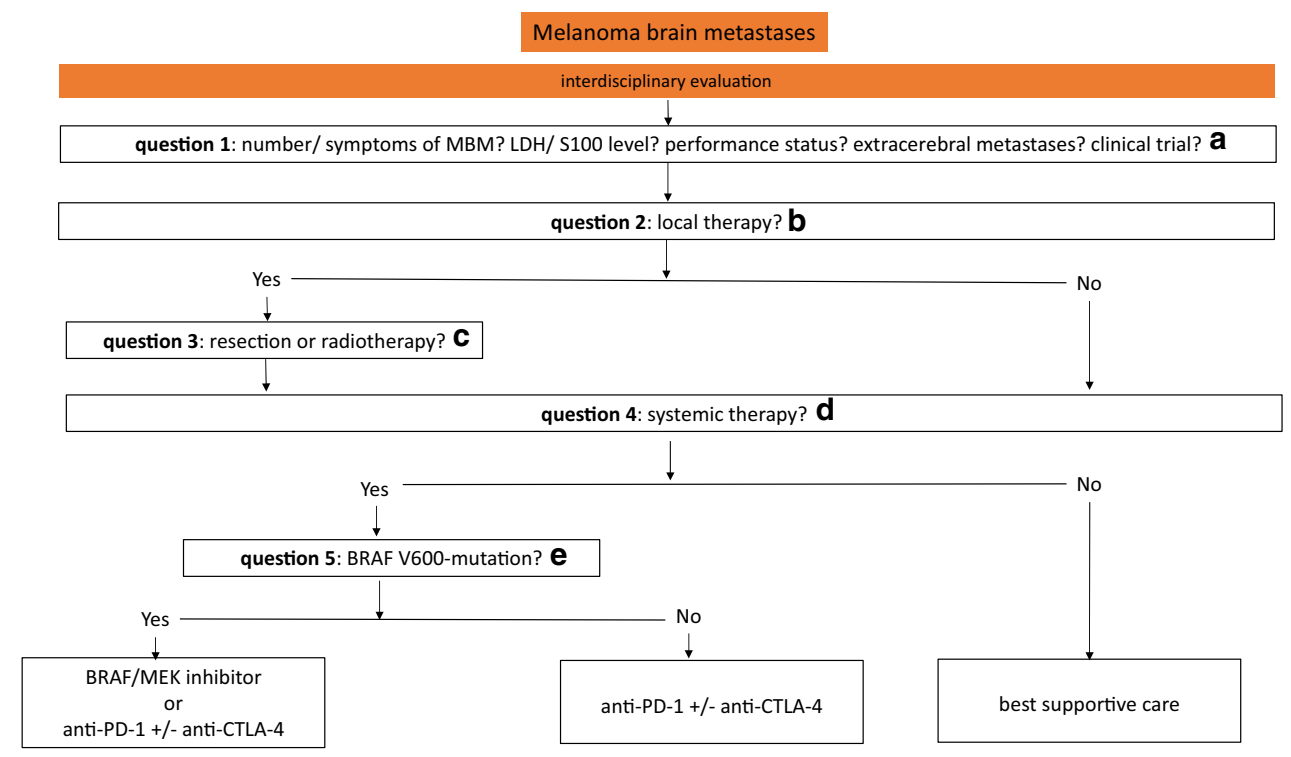

Fig. 1 Algorithm for the management of melanoma brain metastases. Treatment decisions for patients with MBM should be made by an interdisciplinary tumor board in order to establish the best possible treatment for the individual patient. anti-CTLA-4 antibody targeting cytotoxic T-lymphocyte-associated antigen 4 (e.g., ipilimumab), anti$P D-1$ antibody targeting programmed death 1 (e.g., nivolumab, pembrolizumab), $L D H$ lactate dehydrogenase, $M B M$ melanoma brain metastases. a In principle, treatment decisions for patients with MBM should be made in consultation with a multidisciplinary team of neuroradiologists, neurosurgeons, radiation oncologists, and medical oncologists/dermato-oncologists in order to establish the best possible treatment for the individual patient, taking into account the prognostic factors in each case. Enrollment in an appropriate clinical trial is the preferred management for eligible patients. b Upfront local therapies need to be considered, in particular for patients with symptomatic MBM. c The best candidates for neurosurgical resection of brain metastases are patients with solitary or limited brain metastases and adequate condition to undergo surgery. This is particularly the case

\section{Conclusions}

Until recently, brain metastases almost invariably contributed to patients' death, having only limited treatment options. Immune checkpoint inhibitors and BRAF/MEK inhibitors have provided dramatic advances in the treatment of metastatic melanoma. The phase II studies conducted to date have confirmed the efficacy of the new systemic therapies in patients with MBM, and have improved the prognosis for these patients. For checkpoint inhibitors and BRAF/MEK inhibitors, the response rates in the brain are similar to those outside the brain. However, the duration of response appears to be shorter in the brain, particularly for targeted therapy.

The results of retrospective studies suggest that combining stereotactic radiosurgery with immune checkpoint inhibitors or BRAF/MEK inhibitors improves brain metastasis control and prolongs overall survival, without increased toxicity. However, the results of prospective studies are still awaited. when immediate symptom relief is needed. Patients suitable for SRS may have up to 10 metastases with a total cumulative volume $\leq 15 \mathrm{~mL}$. WBRT seems to be a treatment option for patients with multiple MBM who have a high risk of dying of brain metastases. d Best supportive care may be an option for patients with poor general condition and tumors that were refractory to multiple previous therapies. e BRAF mutation status should be assessed before initiating systemic treatment. Although both BRAF/MEK inhibitors and anti-CTLA-4/anti-PD-1 antibodies have shown intracranial activity, the ideal sequencing of these agents in BRAF-mutant MBM is not clear. For patients with BRAF wild-type MBM, immune checkpoint inhibitors are the systemic therapy of choice, in particular nivolumab in combination with ipilimumab, provided that toxicity is considered tolerable for the individual patient. Retrospective reports of combining SRS with immune or targeted therapy appear promising. However, this approach needs to be investigated in prospective studies

Treatment decisions for patients with MBM should be made by an interdisciplinary tumor board in order to establish the best possible treatment for the individual patient, taking into account the prognostic factors in each case.

Though recent studies report encouraging results with intracranial control rates of $50-75 \%$, most patients still progress and die. Thus, prospective studies, particularly of treatment combinations and sequences, innovative treatment strategies, and biomarkers of treatment response in patients with MBM, are urgently needed.

Funding This research received no specific grant from any funding agency in the public, commercial, or not-for-profit sectors.

\section{Compliance with Ethical Standards}

Conflict of interest Dr. Ricarda Rauschenberg received consulting fees from Novartis and GlaxoSmithKline, payments for lectures from Novartis and Amgen, and payments for travel expenses from BristolMyers Squibb. All other authors declare no conflict of interest. 
Open Access This article is distributed under the terms of the Creative Commons Attribution-NonCommercial 4.0 International License (http://creativecommons.org/licenses/by-nc/4.0/), which permits any noncommercial use, distribution, and reproduction in any medium, provided you give appropriate credit to the original author(s) and the source, provide a link to the Creative Commons license, and indicate if changes were made.

\section{References}

1. Nayak L, Lee EQ, Wen PY. Epidemiology of brain metastases. Curr Oncol Rep. 2012;14(1):48-54.

2. Amer $\mathbf{M H}$, et al. Malignant melanoma and central nervous system metastases: incidence, diagnosis, treatment and survival. Cancer. 1978;42(2):660-8.

3. Staudt M, et al. Determinants of survival in patients with brain metastases from cutaneous melanoma. $\mathrm{Br} \mathrm{J}$ Cancer. 2010;102(8):1213-8.

4. Eigentler TK, et al. Number of metastases, serum lactate dehydrogenase level, and type of treatment are prognostic factors in patients with brain metastases of malignant melanoma. Cancer. 2011;117(8):1697-703.

5. Raizer JJ, et al. Brain and leptomeningeal metastases from cutaneous melanoma: survival outcomes based on clinical features. Neuro Oncol. 2008;10(2):199-207.

6. Sperduto PW, et al. Estimating survival in melanoma patients with brain metastases: an update of the graded prognostic assessment for melanoma using molecular markers (melanomamolGPA). Int J Radiat Oncol Biol Phys. 2017;99(4):812-6.

7. Fife KM, et al. Determinants of outcome in melanoma patients with cerebral metastases. J Clin Oncol. 2004;22(7):1293-300.

8. Davies MA, et al. Prognostic factors for survival in melanoma patients with brain metastases. Cancer. 2011;117(8):1687-96.

9. Ajithkumar T, et al. Evolving treatment options for melanoma brain metastases. Lancet Oncol. 2015;16(13):e486-97.

10. Manon R, et al. Phase II trial of radiosurgery for one to three newly diagnosed brain metastases from renal cell carcinoma, melanoma, and sarcoma: an Eastern Cooperative Oncology Group study (E 6397). J Clin Oncol. 2005;23(34):8870-6.

11. Yamamoto M, et al. Stereotactic radiosurgery for patients with multiple brain metastases (JLGK0901): a multi-institutional prospective observational study. Lancet Oncol. 2014;15(4):387-95

12. Skeie BS, et al. Gamma knife surgery in brain melanomas: absence of extracranial metastases and tumor volume strongest indicators of prolonged survival. World Neurosurg. 2011;75(5-6):684-91 (discussion 598-603).

13. Ramakrishna, N. and K.A. Margolin, Multidisciplinary approach to brain metastasis from melanoma; local therapies for central nervous system metastases. Am Soc Clin Oncol Educ Book. 2013;399-403

14. Bernard ME, et al. Linear accelerator based stereotactic radiosurgery for melanoma brain metastases. J Cancer Res Ther. 2012;8(2):215-21.

15. Mingione V, et al. Gamma surgery for melanoma metastases in the brain. J Neurosurg. 2002;96(3):544-51.

16. Hara W, et al. Cyberknife for brain metastases of malignant melanoma and renal cell carcinoma. Neurosurgery. 2009;64(2 Suppl):A26-32.

17. Minniti G, et al. Stereotactic radiosurgery for brain metastases: analysis of outcome and risk of brain radionecrosis. Radiat Oncol. 2011;6:48.

18. Aoyama $\mathrm{H}$, et al. Stereotactic radiosurgery plus whole-brain radiation therapy vs stereotactic radiosurgery alone for treatment of brain metastases: a randomized controlled trial. JAMA. 2006;295(21):2483-91.

19. Shaw E, et al. Single dose radiosurgical treatment of recurrent previously irradiated primary brain tumors and brain metastases: final report of RTOG protocol 90-05. Int J Radiat Oncol Biol Phys. 2000;47(2):291-8.

20. Le Rhun E, et al. Radionecrosis after stereotactic radiotherapy for brain metastases. Expert Rev Neurother. 2016;16(8):903-14.

21. Glitza IC, et al. Bevacizumab as an effective treatment for radiation necrosis after radiotherapy for melanoma brain metastases. Melanoma Res. 2017;27(6):580-584

22. Levin VA, et al. Randomized double-blind placebo-controlled trial of bevacizumab therapy for radiation necrosis of the central nervous system. Int J Radiat Oncol Biol Phys. 2011;79(5):1487-95.

23. de la Fuente M, et al. Whole-brain radiotherapy in patients with brain metastases from melanoma. CNS Oncol. 2014;3(6):401-6.

24. Soffietti R, et al. A European Organisation for Research and Treatment of Cancer phase III trial of adjuvant whole-brain radiotherapy versus observation in patients with one to three brain metastases from solid tumors after surgical resection or radiosurgery: quality-of-life results. J Clin Oncol. 2013;31(1):65-72.

25. Corn BW, et al. Prospective evaluation of quality of life and neurocognitive effects in patients with multiple brain metastases receiving whole-brain radiotherapy with or without thalidomide on Radiation Therapy Oncology Group (RTOG) trial 0118. Int J Radiat Oncol Biol Phys. 2008;71(1):71-8.

26. Tsao MN, et al. Whole brain radiotherapy for the treatment of newly diagnosed multiple brain metastases. Cochrane Database Syst Rev. 2012;(4):CD003869.

27. Teng F, et al. Blood-tumor barrier opening changes in brain metastases from pre to one-month post radiation therapy. Radiother Oncol. 2017;125(1):89-93.

28. Gondi V, et al. Preservation of memory with conformal avoidance of the hippocampal neural stem-cell compartment during whole-brain radiotherapy for brain metastases (RTOG 0933): a phase II multi-institutional trial. J Clin Oncol. 2014;32(34):3810-6.

29. Mahajan A, et al. Post-operative stereotactic radiosurgery versus observation for completely resected brain metastases: a singlecentre, randomised, controlled, phase 3 trial. Lancet Oncol. 2017;18(8):1040-8.

30. Lamba N, et al. Stereotactic radiosurgery versus whole-brain radiotherapy after intracranial metastasis resection: a systematic review and meta-analysis. Radiat Oncol. 2017;12(1):106.

31. Kocher $\mathbf{M}$, et al. Adjuvant whole-brain radiotherapy versus observation after radiosurgery or surgical resection of one to three cerebral metastases: results of the EORTC 22952-26001 study. J Clin Oncol. 2011;29(2):134-41.

32. Patchell RA, et al. Postoperative radiotherapy in the treatment of single metastases to the brain: a randomized trial. JAMA. 1998;280(17):1485-9.

33. Mori Y, et al. Stereotactic radiosurgery for cerebral metastatic melanoma: factors affecting local disease control and survival. Int J Radiat Oncol Biol Phys. 1998;42(3):581-9.

34. Davies H, et al. Mutations of the BRAF gene in human cancer. Nature. 2002;417(6892):949-54.

35. Long GV, et al. Dabrafenib and trametinib versus dabrafenib and placebo for Val600 BRAF-mutant melanoma: a multicentre, double-blind, phase 3 randomised controlled trial. Lancet. 2015;386(9992):444-51.

36. Larkin MG, Yan Y, McArthur GA et al. Update of progressionfree survival (PFS) and correlative biomarker analysis from coBRIM: phase III study of cobimetinib (cobi) plus vemurafenib (vem) in advanced BRAF-mutated melanoma. J Clin Oncol. 2015;33(suppl):abstr. 9006. 
37. Ascierto PA, et al. Cobimetinib combined with vemurafenib in advanced BRAF(V600)-mutant melanoma (coBRIM): updated efficacy results from a randomised, double-blind, phase 3 trial. Lancet Oncol. 2016;17(9):1248-60.

38. Long GV, et al. Dabrafenib in patients with Val600Glu or Val600Lys BRAF-mutant melanoma metastatic to the brain (BREAK-MB): a multicentre, open-label, phase 2 trial. Lancet Oncol. 2012;13(11):1087-95.

39. McArthur GA, et al. Vemurafenib in metastatic melanoma patients with brain metastases: an open-label, single-arm, phase 2, multicentre study. Ann Oncol. 2017;28(3):634-41.

40. Davies MA, et al. Dabrafenib plus trametinib in patients with BRAFV600-mutant melanoma brain metastases (COMBI-MB): a multicentre, multicohort, open-label, phase 2 trial. Lancet Oncol. 2017;18(7):863-73.

41. Peuvrel L, et al. Incidence and characteristics of melanoma brain metastases developing during treatment with vemurafenib. J Neurooncol. 2014;120(1):147-54.

42. Amaral T, et al. The mitogen-activated protein kinase pathway in melanoma part I-activation and primary resistance mechanisms to BRAF inhibition. Eur J Cancer. 2017;73:85-92.

43. Dunn GP, Old LJ, Schreiber RD. The three Es of cancer immunoediting. Annu Rev Immunol. 2004;22:329-60.

44. Davar D, et al. High-dose interleukin-2 (HD IL-2) for advanced melanoma: a single center experience from the University of Pittsburgh Cancer Institute. J Immunother Cancer. 2017;5(1):74.

45. Rosenberg SA, et al. Treatment of 283 consecutive patients with metastatic melanoma or renal cell cancer using high-dose bolus interleukin 2. JAMA. 1994;271(12):907-13.

46. Ribas A. Tumor immunotherapy directed at PD-1. N Engl J Med. 2012;366(26):2517-9.

47. Larkin J, et al. Combined nivolumab and ipilimumab or monotherapy in untreated melanoma. $\mathrm{N}$ Engl $\mathrm{J}$ Med. 2015:373(1):23-34.

48. Wolchok JD, et al. Overall Survival with Combined Nivolumab and Ipilimumab in Advanced Melanoma. $\mathrm{N}$ Engl $\mathrm{J}$ Med. 2017;377(14):1345-1356

49. Margolin K, et al. Ipilimumab in patients with melanoma and brain metastases: an open-label, phase 2 trial. Lancet Oncol. 2012;13(5):459-65.

50. Goldberg SB, et al. Pembrolizumab for patients with melanoma or non-small-cell lung cancer and untreated brain metastases: early analysis of a non-randomised, open-label, phase 2 trial. Lancet Oncol. 2016;17(7):976-83.

51. Long GV, Atkinson V, Menzies AM, Lo S, Guminski A, Brown MP, Gonzales M, Diamante K, Sandhu S, Scolyer RA, Emmett $\mathrm{L}, \mathrm{Mc}$ Arthur GA. A randomized phase II study of nivolumab or nivolumab combined with ipilimumab in patients (pts) with melanoma brain metastases (mets): the Anti-PD1 Brain Collaboration (ABC). J Clin Oncol, 2017;35(15 suppl):9508.

52. Hugo W, et al. Non-genomic and Immune Evolution of Melanoma Acquiring MAPKi Resistance. Cell. 2015;162(6):1271-85.

53. Tawbi H.A.H, et al. Efficacy and safety of nivolumab (NIVO) plus ipilimumab (IPI) in patients with melanoma (MEL) metastatic to the brain: results of the phase II study checkMate 204. J Clin Oncol. 2017;35(suppl):abstr. 9507.

54. Rauschenberg R, et al. Melanoma brain metastases: treatment options. Hautarzt. 2016;67(7):536-43.

55. Ryken TC, et al. The role of steroids in the management of brain metastases: a systematic review and evidence-based clinical practice guideline. J Neurooncol. 2010;96(1):103-14.

56. Mikkelsen $\mathrm{T}$, et al. The role of prophylactic anticonvulsants in the management of brain metastases: a systematic review and evidence-based clinical practice guideline. J Neurooncol. 2010;96(1):97-102.
57. Rekers NH, et al. Stereotactic ablative body radiotherapy combined with immunotherapy: present status and future perspectives. Cancer Radiother. 2014;18(5-6):391-5.

58. Demaria S, Formenti SC. Role of T lymphocytes in tumor response to radiotherapy. Front Oncol. 2012;2:95.

59. Mole RH. Whole body irradiation; radiobiology or medicine? $\mathrm{Br}$ J Radiol. 1953;26(305):234-41.

60. Reynders K, et al. The abscopal effect of local radiotherapy: using immunotherapy to make a rare event clinically relevant. Cancer Treat Rev. 2015;41(6):503-10.

61. Vanpouille-Box C, et al. In situ vaccination by radiotherapy to improve responses to anti-CTLA-4 treatment. Vaccine. 2015;33(51):7415-22.

62. Demaria $\mathrm{S}$, et al. Ionizing radiation inhibition of distant untreated tumors (abscopal effect) is immune mediated. Int $\mathbf{J}$ Radiat Oncol Biol Phys. 2004;58(3):862-70.

63. Chakravarty PK, et al. Flt3-ligand administration after radiation therapy prolongs survival in a murine model of metastatic lung cancer. Cancer Res. 1999;59(24):6028-32.

64. Dewan MZ, et al. Fractionated but not single-dose radiotherapy induces an immune-mediated abscopal effect when combined with anti-CTLA-4 antibody. Clin Cancer Res. 2009;15(17):5379-88.

65. Stamell EF, et al. The abscopal effect associated with a systemic anti-melanoma immune response. Int J Radiat Oncol Biol Phys. 2013;85(2):293-5.

66. Hiniker SM, et al. A systemic complete response of metastatic melanoma to local radiation and immunotherapy. Transl Oncol. 2012;5(6):404-7.

67. Postow MA, et al. Immunologic correlates of the abscopal effect in a patient with melanoma. $\mathrm{N}$ Engl $\mathrm{J}$ Med. 2012;366(10):925-31.

68. Sambade MJ, et al. Melanoma cells show a heterogeneous range of sensitivity to ionizing radiation and are radiosensitized by inhibition of B-RAF with PLX-4032. Radiother Oncol. 2011;98(3):394-9.

69. Hecht M, et al. Radiosensitization by BRAF inhibitor therapymechanism and frequency of toxicity in melanoma patients. Ann Oncol. 2015;26(6):1238-44.

70. Choong ES, et al. Survival of patients with melanoma brain metastasis treated with stereotactic radiosurgery and active systemic drug therapies. Eur J Cancer. 2017;75:169-78.

71. Gaudy-Marqueste C, et al. Survival of melanoma patients treated with targeted therapy and immunotherapy after systematic upfront control of brain metastases by radiosurgery. Eur $\mathrm{J}$ Cancer. 2017;84:44-54.

72. Williams NL, et al. Phase 1 study of ipilimumab combined with whole brain radiation therapy or radiosurgery for melanoma patients with brain metastases. Int $\mathbf{J}$ Radiat Oncol Biol Phys. 2017;99(1):22-30.

73. Ahmed KA, et al. Clinical outcomes of melanoma brain metastases treated with stereotactic radiation and anti-PD-1 therapy. Ann Oncol. 2016;27(3):434-41.

74. Patel BG, et al. Initial experience with combined BRAF and MEK inhibition with stereotactic radiosurgery for BRAF mutant melanoma brain metastases. Melanoma Res. 2016;26(4):382-6.

75. Kotecha R, et al. Melanoma brain metastasis: the impact of stereotactic radiosurgery, BRAF mutational status, and targeted and/or immune-based therapies on treatment outcome. J Neurosurg. 2017:1-10.

76. Peuvrel L, et al. Severe radiotherapy-induced extracutaneous toxicity under vemurafenib. Eur J Dermatol. 2013;23(6):879-81.

77. Gaudy-Marqueste C, et al. On demand Gamma-Knife strategy can be safely combined with BRAF inhibitors for the treatment of melanoma brain metastases. Ann Oncol. 2014;25(10):2086-91. 
78. Ly D, et al. Local control after stereotactic radiosurgery for brain metastases in patients with melanoma with and without BRAF mutation and treatment. J Neurosurg. 2015;123(2):395-401.

79. Kohutek ZA, et al. Long-term risk of radionecrosis and imaging changes after stereotactic radiosurgery for brain metastases. J Neurooncol. 2015;125(1):149-56.

80. Colaco RJ, et al. Does immunotherapy increase the rate of radiation necrosis after radiosurgical treatment of brain metastases? J Neurosurg. 2016;125(1):17-23.

81. Mathew M, et al. Ipilimumab in melanoma with limited brain metastases treated with stereotactic radiosurgery. Melanoma Res. 2013;23(3):191-5.

82. Silk AW, et al. Ipilimumab and radiation therapy for melanoma brain metastases. Cancer Med. 2013;2(6):899-906.

83. Anker CJ, et al. Avoiding severe toxicity from combined braf inhibitor and radiation treatment: consensus guidelines from the Eastern Cooperative Oncology Group (ECOG). Int J Radiat Oncol Biol Phys. 2016;95(2):632-46.

84. Dovedi SJ, et al. Acquired resistance to fractionated radiotherapy can be overcome by concurrent PD-L1 blockade. Cancer Res. 2014;74(19):5458-68.

85. Qian JM, et al. Timing and type of immune checkpoint therapy affect the early radiographic response of melanoma brain metastases to stereotactic radiosurgery. Cancer. 2016;122(19):3051-8.

86. Chajon E, et al. The synergistic effect of radiotherapy and immunotherapy: A promising but not simple partnership. Crit Rev Oncol Hematol. 2017;111:124-32.

87. Smalley KS, et al. Managing leptomeningeal melanoma metastases in the era of immune and targeted therapy. Int $\mathrm{J}$ Cancer. 2016;139(6):1195-201.

88. Pape E, et al. Leptomeningeal metastasis in melanoma: a prospective clinical study of nine patients. Vivo. 2012;26(6):1079-86.

89. Harstad L, Hess KR, Groves MD. Prognostic factors and outcomes in patients with leptomeningeal melanomatosis. Neuro Oncol. 2008;10(6):1010-8.

90. Le Rhun E, Taillibert S, Chamberlain MC. Carcinomatous meningitis: Leptomeningeal metastases in solid tumors. Surg Neurol Int. 2013;4(Suppl 4):S265-88.
91. Gil B, et al. Detection of leptomeningeal metastasis by contrastenhanced 3D T1-SPACE: comparison with 2D FLAIR and contrast-enhanced 2D T1-weighted images. PLoS One. 2016;11(10):e0163081.

92. Glantz MJ, et al. Cerebrospinal fluid cytology in patients with cancer: minimizing false-negative results. Cancer. 1998;82(4):733-9.

93. Chamberlain M, et al. Leptomeningeal metastases: a RANO proposal for response criteria. Neuro Oncol. 2017;19(4):484-92.

94. Lee JM, et al. Long-term stabilization of leptomeningeal disease with whole-brain radiation therapy in a patient with metastatic melanoma treated with vemurafenib: a case report. Melanoma Res. 2013;23(2):175-8.

95. Floudas CS, Chandra AB, Xu Y. Vemurafenib in leptomeningeal carcinomatosis from melanoma: a case report of near-complete response and prolonged survival. Melanoma Res. 2016;26(3):312-5.

96. Bot I, Blank CU, Brandsma D. Clinical and radiological response of leptomeningeal melanoma after whole brain radiotherapy and ipilimumab. J Neurol. 2012;259(9):1976-8.

97. Glitza IC, Ferguson SD, Guha-Thakurta N. Rapid resolution of leptomeningeal disease with targeted therapy in a metastatic melanoma patient. J Neurooncol. 2017;133(3):663-5.

98. Geukes Foppen MH, et al. Targeted treatment and immunotherapy in leptomeningeal metastases from melanoma. Ann Oncol. 2016;27(6):1138-42.

99. Arasaratnam M, et al. Leptomeningeal melanoma-A case series in the era of modern systemic therapy. Pigment Cell Melanoma Res. 2018;31(1):120-124

100. Papadopoulos NE, Gerber DL, Eton O. The role of intrathecal (IT) use of interleukin-2 (IL-2) in the treatment of leptomeningeal disease (LMD) in patients (pts) with melanoma. Proc Am Soc Clin Oncol. 2002;21:(abstr 1408).

101. Shonka NA, Kessinger AM, Aizenberg MR. Intrathecal interleukin-2 for melanomatous meningitis. $\mathrm{J}$ Clin Oncol. 2014;32(33):e111-3.

102. Glitza IC, et al. Intrathecal administration of tumor-infiltrating lymphocytes is well tolerated in a patient with leptomeningeal disease from metastatic melanoma: a case report. Cancer Immunol Res. 2015;3(11):1201-6. 